\title{
Double Brillouin frequency shift through circulation of odd-order Stokes signal
}

\begin{abstract}
We demonstrate a simple configuration for generating a double Brillouin frequency shift through the circulation of an odd-order Brillouin Stokes signal. It is operated based on cascaded Brillouin scattering in single-mode optical fibers that behave as the Brillouin gain media. A four-port circulator is incorporated into the setup to circulate the odd-order Brillouin Stokes signal in the fiber. It thus initiates a higher order Brillouin Stokes signal, which is double Brillouin frequency downshifted from the input signal. For the $5 \mathrm{Wkm}$ long

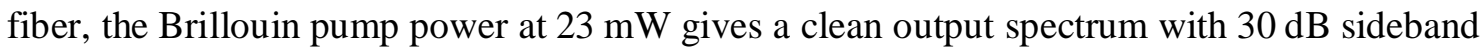
suppression ratio. The output signal is $0.174 \mathbb{7 m}$ or $\sim 21.7 \mathbb{C H} \mathrm{Hz}$ downshifted from the input signal.
\end{abstract}

